\title{
Superresolution microscopy with transient binding
}

\author{
Julia Molle ${ }^{{ }^{1}}$, Mario Raab* ${ }^{1}$, Susanne Holzmeister ${ }^{1}$, Daniel Schmitt-Monreal ${ }^{1}$, Dina Grohmann ${ }^{2}$, Zhike He ${ }^{3}$ and \\ Philip Tinnefeld ${ }^{1}$ \\ ${ }^{1}$ Technische Universität Braunschweig, Institut für Physikalische und Theoretische Chemie-NanoBioSciences, \\ and Braunschweig Integrated Centre of Systems Biology (BRICS), and Laboratory for Emerging Nanometrology
}

(LENA), Braunschweig University of Technology, Hans-Sommer-Straße 10, 38106 Braunschweig, Germany.

${ }^{2}$ Universität Regensburg, Institut für Biochemie, Genetik und Mikrobiologie, Lehrstuhl für Mikrobiologie, Universitätsstraße 31, 93053 Regensburg

${ }^{3}$ Key Laboratory of Analytical Chemistry for Biology and Medicine (Ministry of Education), College of Chemistry and Molecular Sciences, Wuhan University, Wuhan 430072, P. R. China

p.tinnefeld@tu-braunschweig.de

*these authors contributed equally

\begin{abstract}
:
For single-molecule localization based superresolution, the concentration of fluorescent labels has to be thinned out. This is commonly achieved by photophysically or photochemically deactivating subsets of molecules. Alternatively, apparent switching of molecules can be achieved by transient binding of fluorescent labels. Here, a diffusing dye yields bright fluorescent spots when binding to the structure of interest. As the binding interaction is weak, the labeling is reversible and the dye ligand construct diffuses back into solution. This approach of achieving Superresolution by Transient Binding (STB) is reviewed in this manuscript. Different realizations of STB are discussed and compared to other localization-based superresolution modalities. We propose the development of labeling strategies that will make STB a highly versatile tool for superresolution microscopy at highest resolution.
\end{abstract}

\section{Introduction:}

Abbé's law determined the resolution limit of far-field light microscope to be approximately half the wavelength of light [1]. Implementations of different concepts enabled fluorescence microscopy with nanoscale resolution, and therefore making the diffraction barrier a thing of the past. The essential element in resolving fluorescence objects closer together than $200 \mathrm{~nm}$ was to switch their signals successively on and off. The two prevalent concepts for superresolution microscopy distinguish themselves by using targeted readout (e.g. STED microscopy [2]) or stochastic readout (including (F)PALM and (d)STORM)[3-6]. Superresolution based on stochastic switching and readout implies that the majority of molecules are prepared in an off-state and the population fluorescent molecules is so sparse that each molecule can be localized by fitting an appropriate 
function to their isolated point-spread-function on the acquired camera images [3-5]. Different realizations of this approach were given different acronyms that historically distinguished themselves by the way switching was achieved. In the first realization, for example, photoactivatable fluorescent proteins were switched on with dim UV light while recording the upcoming single-molecule events [3]. Next, the dicarbocyanine Cy5 was switched on- and off- in the presence of thiols and optionally an activator dye such as Cy3 $[4,6]$. Ways to switch single molecules for this sort of stochastic switching and readout were reviewed recently [7-10]. In the meantime, it has been realized that reversible switching of molecules can be disadvantageous because the total photon budget of a molecule is distributed in different switching cycles, reducing the localization precision per localization event. Additionally, reversible switching can be detrimental in approaches for counting molecules. On the other hand, multiple switching cycles increase the probability that molecules are not missed. The early approaches using photoinduced switching have several short-comings in that the photon budget is limited, the choice of dyes is limited, the number of ON-counts and thereby the localization precision is limited as is the multiplexing ability.

In 2006, however, a non-photochemical approach to thinning out fluorescent labels for superresolution was presented by the Hochstrasser lab and termed PAINT (Points Accumulation for Imaging in Nanoscale Topography) [11]. The technique relies on stochastic binding of diffusing fluorescent probes that are continuously localized once they bind to an object of interest. If then only one fluorophore within a diffraction limited radius is in the 'on-state', it can be localized with high accuracy using a two-dimensional Gaussian fitting. Unbinding or photobleaching then terminates the localization event and repetition of the process allows reconstruction of superresolved images. The principle of PAINT is illustrated in Figure 1.
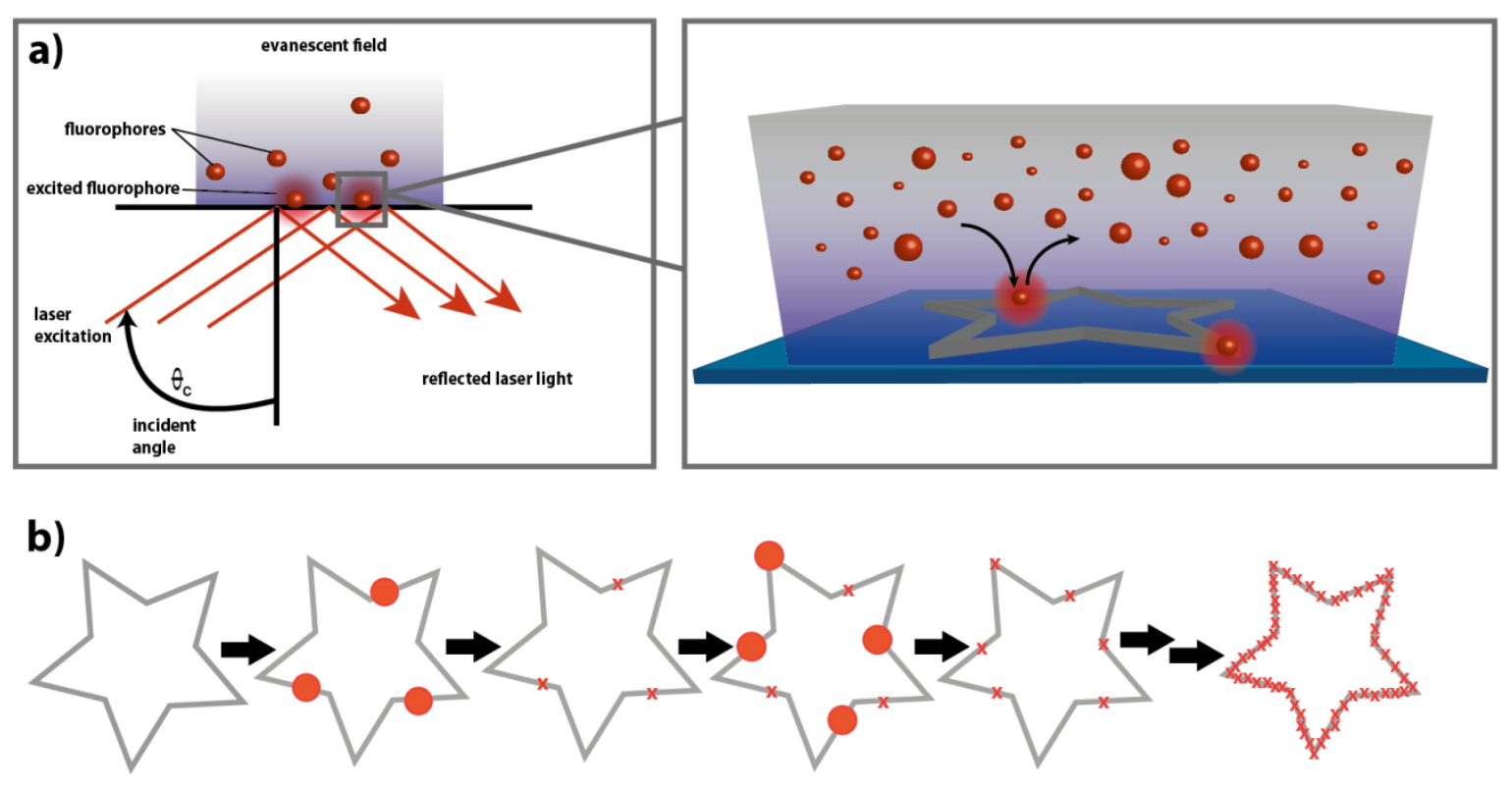

Figure 1 The principle of STB: Breaking the diffraction limit is reached by stochastic and transient binding and subsequent localization. a) In contrast to other methods the apparent blinking is reached by the diffusing fluorophores binding stochastically to the structure of interest. Light-sheet or TIRF-illumination is required to ensure the necessary signal-to-noise ratio for single-molecule detection. b) Fluorophores binding to the target structure (here a "star") are localized via 2D Gaussian fitting. Switching off the single-molecule fluorescence is achieved by unbinding or bleaching. 


\section{Implementation of superresolution by transient binding (STB)}

STB was first demonstrated by imaging lipid bilayers and large unilamellar vesicles [11]. A lipophilic dye (Nile Red) which did not emit fluorescence in aqueous solution but within hydrophobic environments, in this case especially when bound to the lipid core was used (see Figure 2).
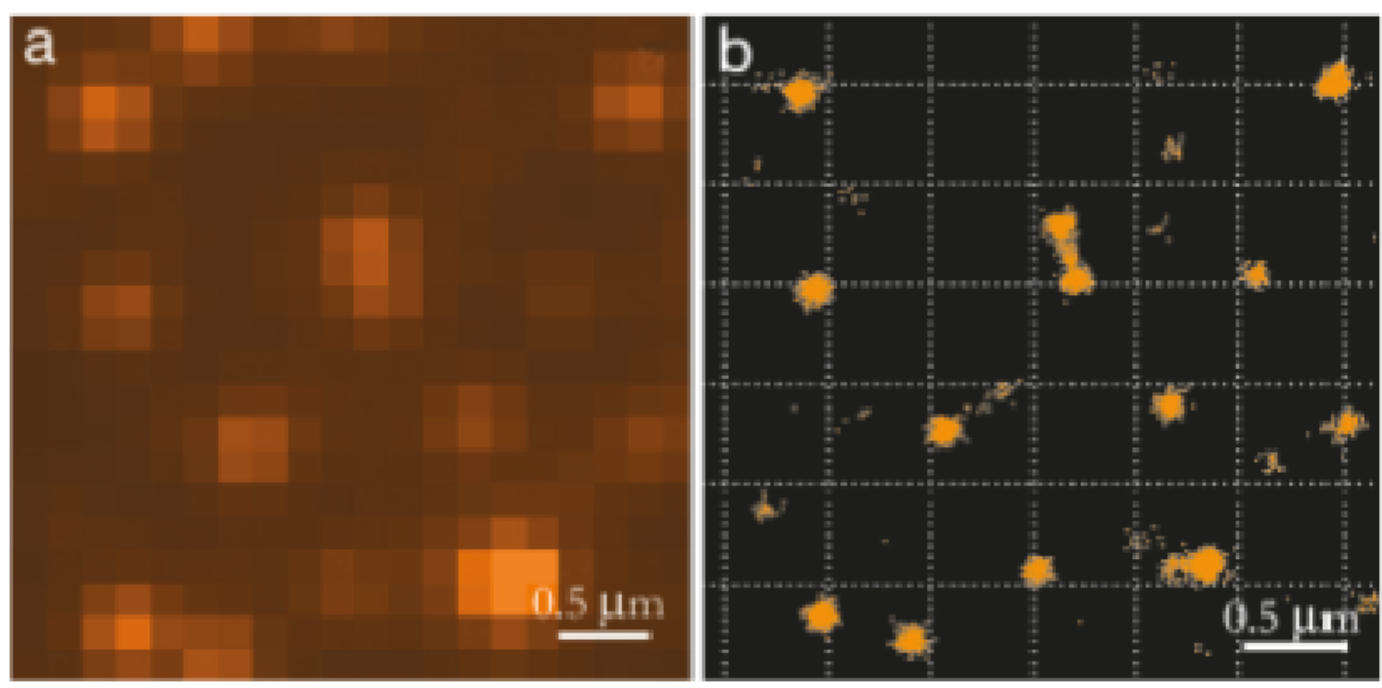

Figure 2 Diffraction limited TIRF image (a) and superresolution PAINT image (b) of unilamellar vesicles attached to a glass surface (Figure adapted from ref. [11] with permission).

In fact, Nile Red was not sensitive enough to the phase of the lipid, so other possible probes for PAINT images of lipid phase domains were investigated. Finally Merocyanine 540 was considered useful for imaging phase domains in binary lipids [12]. Moreover, PAINT with Nile Red was used for a 2 color-experiment in combination with photoinduced eYFP-blinking by Lew et al. to achieve super resolution images of a cell surface [13].

Since the binding rate in STB linearly depends on the probes' concentration, binding can easily be controlled $[11,14]$. Other advantages of STB can be that the object itself is not permanently labeled (at least in the case of PAINT), and the fluorophores are permanently exchanged. Hence, the problem of photobleaching can be circumvented and a high photon budget can be reached because all photons before photobleaching of a binding dye can be used for one localization. Additionally, there are almost no constraints for the selection of dyes and the brightest and most photostable dyes can be used. A drawback of STB is the high number of fluorophores in solution so imaging with epi-fluorescence would lead to an enormous background signal. Therefore techniques that only illuminate the imaging plane like total internal reflection fluorescence (TIRF) or light sheet microscopy (or HILO) are mandatory $[15,16]$. Because PAINT is not adapted for imaging specific molecules, it did not find many biological applications until 2010 when new methods entered this field that extended the STB-principle by specific transient binding $[14,17]$.

\section{DNA-PAINT}

An important technique that combines PAINT with specific binding is DNA-PAINT, established in 2010 [14]. Instead of labeling the structure of interest with a fluorescent dye, it is labeled with a short DNA sequence (docking strand). The complementary dye labeled sequence (imager strand) is applied in the solution to visualize the target structure by hybridization. Transient binding emulating blinking is ensured by using short sequences of 8-10 nucleotides yielding binding times in the milliseconds to second range. An additional feature is the fact that the imager strands are coiled in solution and therefore the fluorescence of dyes such as ATT0655 is substantially quenched by guanine-bases, which leads to a significant background reduction [18]. The technique was demonstrated on DNA origami nanostructures that have been shown to be ideal test structures for superresolution microscopy [19-23]. The principle of DNA PAINT and an example taken from the original publication are shown in Figure 3. 

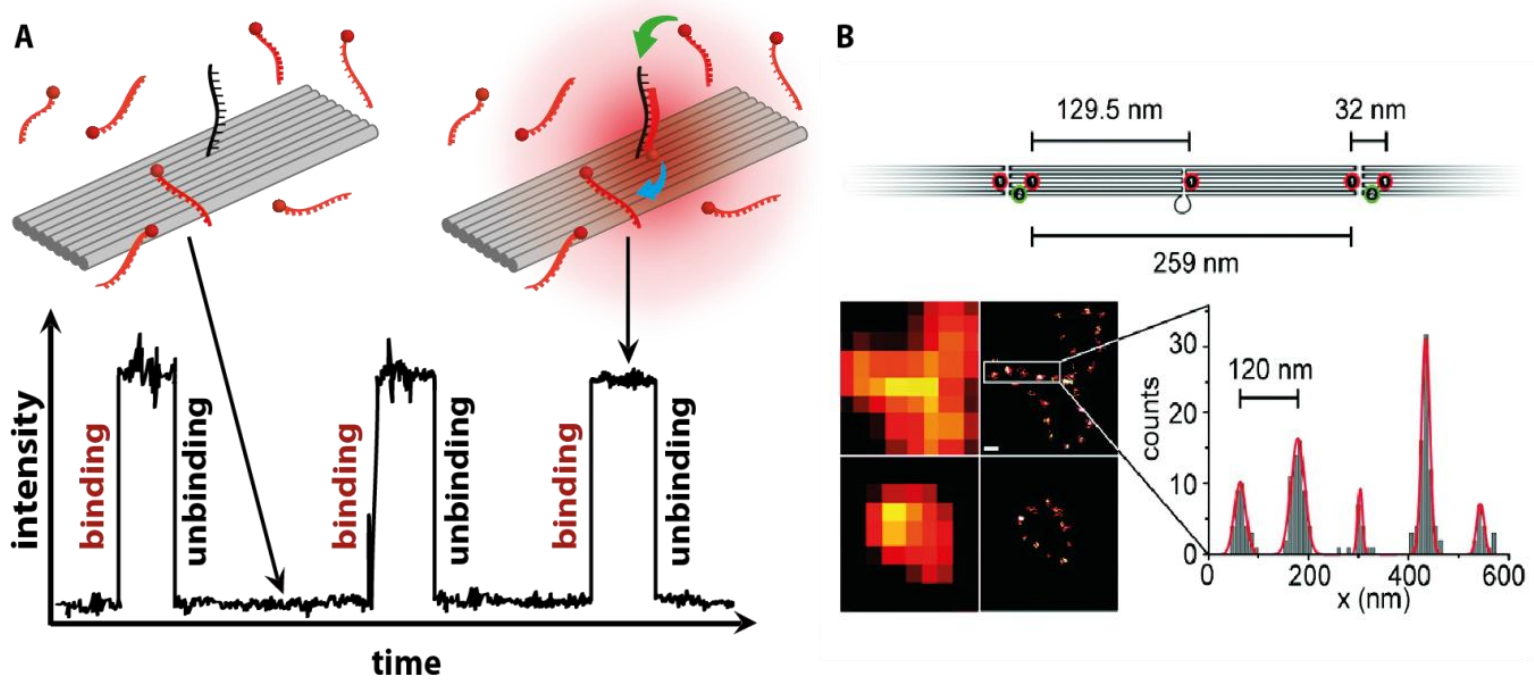

\section{time}

Figure 3 The principle of DNA PAINT: STB is obtained by the binding of short dye-labeled DNA-strands (imager strands) to complementary strands (docking strands) on the structure of interest (A). This type of imaging was first presented on DNA-origami-nanostructures such as a polymer of a long rectangular DNA origami (B) (scale bar, $120 \mathrm{~nm}$ ) [14].

The highly specific binding could be clearly shown and was also confirmed quantitatively by Chen et al. [24]. The usage of DNA-PAINT developed especially in the field of DNA-Nanotechnology [25], e.g. to analyze the structure of polyhedral DNA nanostructures that were difficult to characterize by other means such as electron microscopy. An example of 3D superresolution of a tetrahedral DNA origami that also qualifies as a 3D test sample is presented in Figure $4[25,26]$. DNA PAINT has also been used for the quantitative structural analysis of DNA-nanorods made as a synthetic cargo for motor proteins [27], and to visualize a conjugated polymer routed on a DNA origami template [28]. In 2012 Lin et al. used DNA PAINT for reading out fluorescent barcodes that are smaller than the diffraction limit. This was done in a three-color experiment, showing that DNA-PAINT is applicable for different dyes and thus for multicolor imaging [29,30]. Johnson-Buck et al. used 2-color-DNAPAINT to follow enzyme catalyzed DNA-cleavage over time and to show structural inhomogenities of DNA origami structures [31]. They further compared the usage of DNA PAINT and single particle FRET for the investigation of DNA hybridization kinetics [32,33]. Another work by Scheible and coworkers describes the analysis of DNA origami structures positioned on a lithographic gold array by DNA PAINT [34].

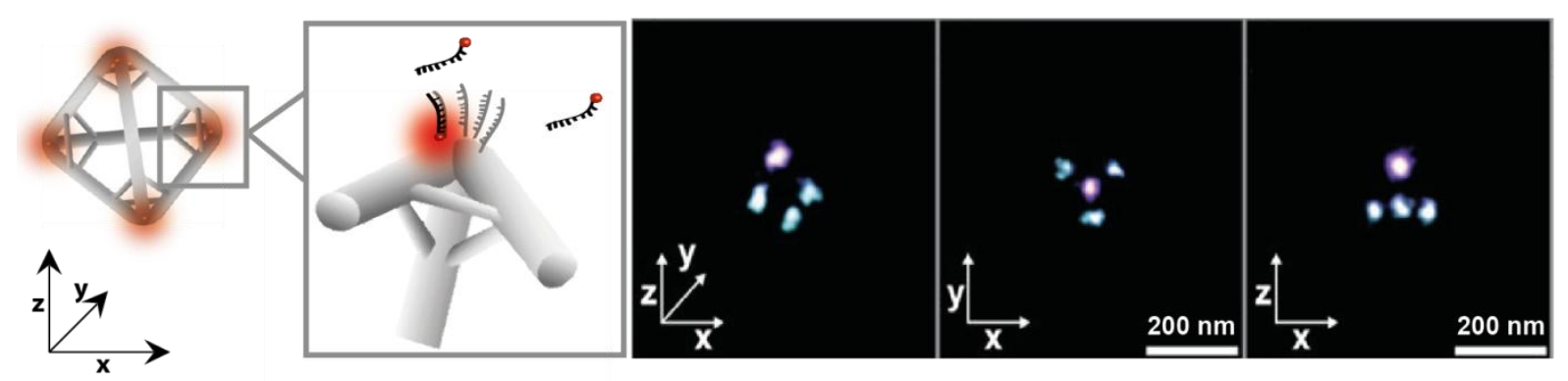

Figure 4 Tetrahedral DNA-Origami-structures imaged with 3D-DNA-PAINT. The vertices of the nanostructures were equipped with multiple docking strands. The side length is $100 \mathrm{~nm}$. Images adapted with permission from [25].

A special ability of DNA PAINT is its multiplexing potential owing to the selectivity of DNA hybridization. Jungmann et al recently presented the pseudo-multicolor-imaging-method EXCHANGE-PAINT that makes use of the fact that the imager-sequence in DNA-PAINT can be exchanged between two measurements just by changing the imaging-buffer [35]. The principle is illustrated in Figure 5. The advantage of Exchange-PAINT is that each "color"-channel can be imaged with the same dye and the same laser line. Thus, the different color channels don't need to be corrected for chromatic aberrations as in usual multicolor imaging and there is no 
risk of spectral crosstalk. This principle can also be used in STED-microscopy as shown in a 4-color measurement by Beater et al. [36].
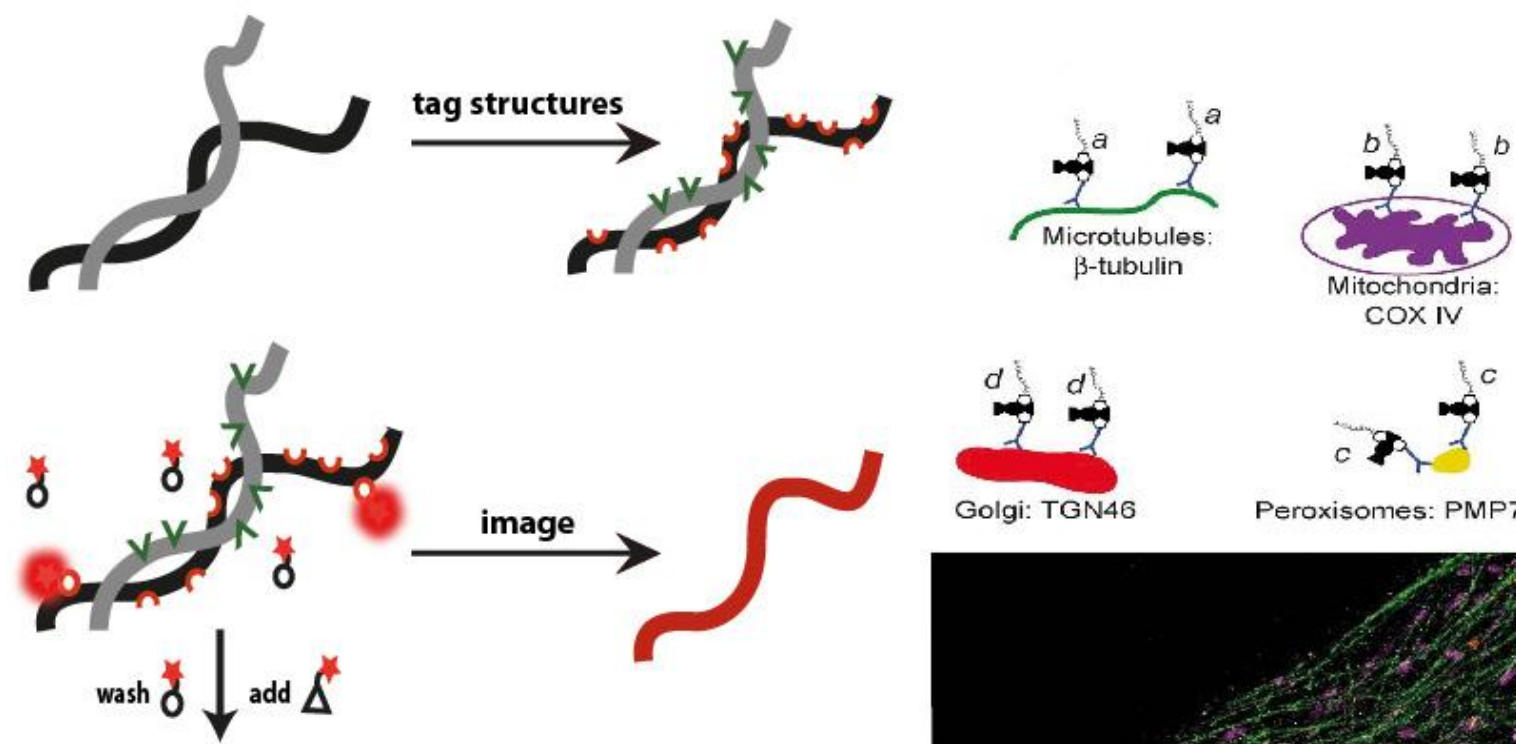

Golgi: TGN46

Peroxisones: PMP70
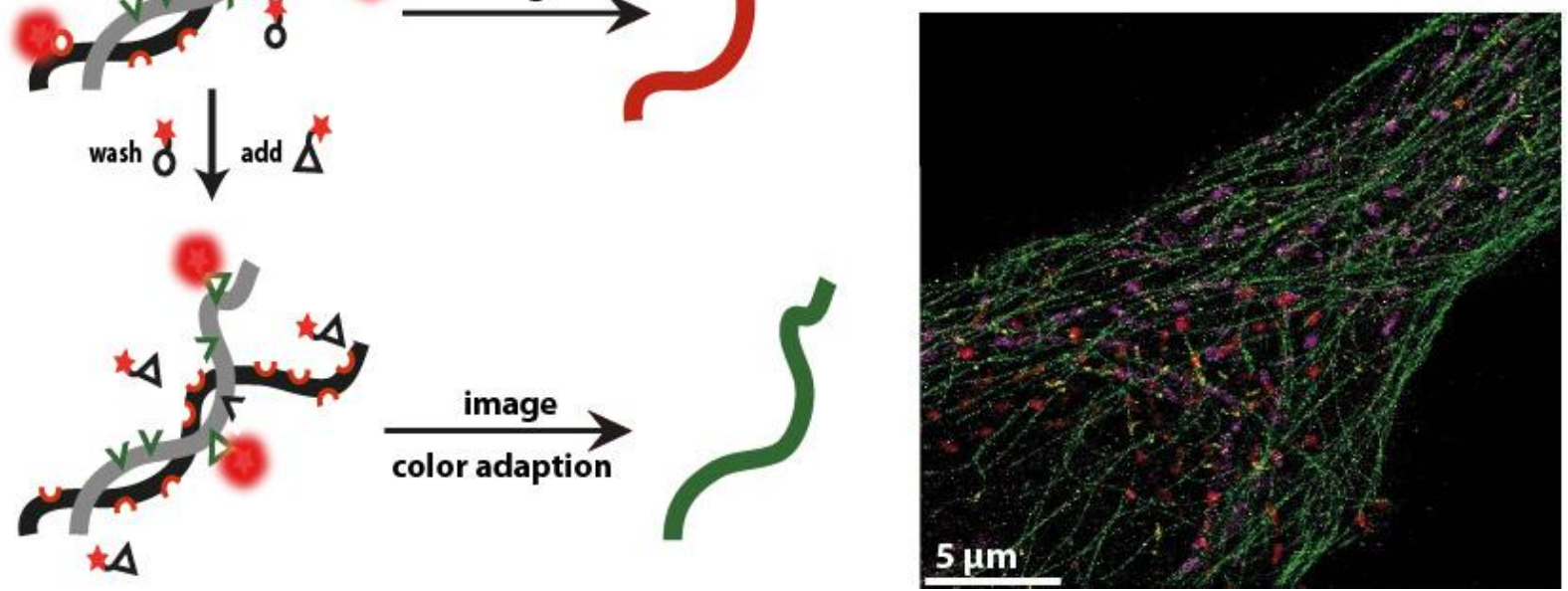

Figure 5 Multiplexing with EXCHANGE-PAINT: different colors are not obtained by using different laser lines but by labeling different structures with different DNA sequences that are visualized by exchanging the imager sequences between the measurements (A). This technique can e.g. be used to make a 4-color-cell image (B) (adapted from [35] with permission.

With the aid of DNA labeled antibodies, Jungmann and coworkers also showed that DNA-PAINT is not only applicable for the imaging of DNA-nanostructures but also for biological samples like fixed cells. For this purpose they labeled antibodies with DNA-binding-sites via biotin-streptavidin binding [35]. Another extension of DNA PAINT to biological samples was realized by Beliveau et al. [37]. In this work chromosomes were labelled with partially complementary oligos that are synthesized and extended with DNA-PAINT binding sites using a PCR-based method $[37,38]$. 
Important features of DNA-PAINT are the high photon numbers that can be reached by bleaching dyes before they unbind the docking site. It has been shown that measuring DNA PAINT at high integration times (300 ms) enables resolving two neighboring docking strands at a distance of only $6 \mathrm{~nm}$, as shown in Figure 6 [39]. Using the full photon budget in combination with more sophisticated stabilization and correction schemes will allow a resolution down to the physical size of the dye labels used.

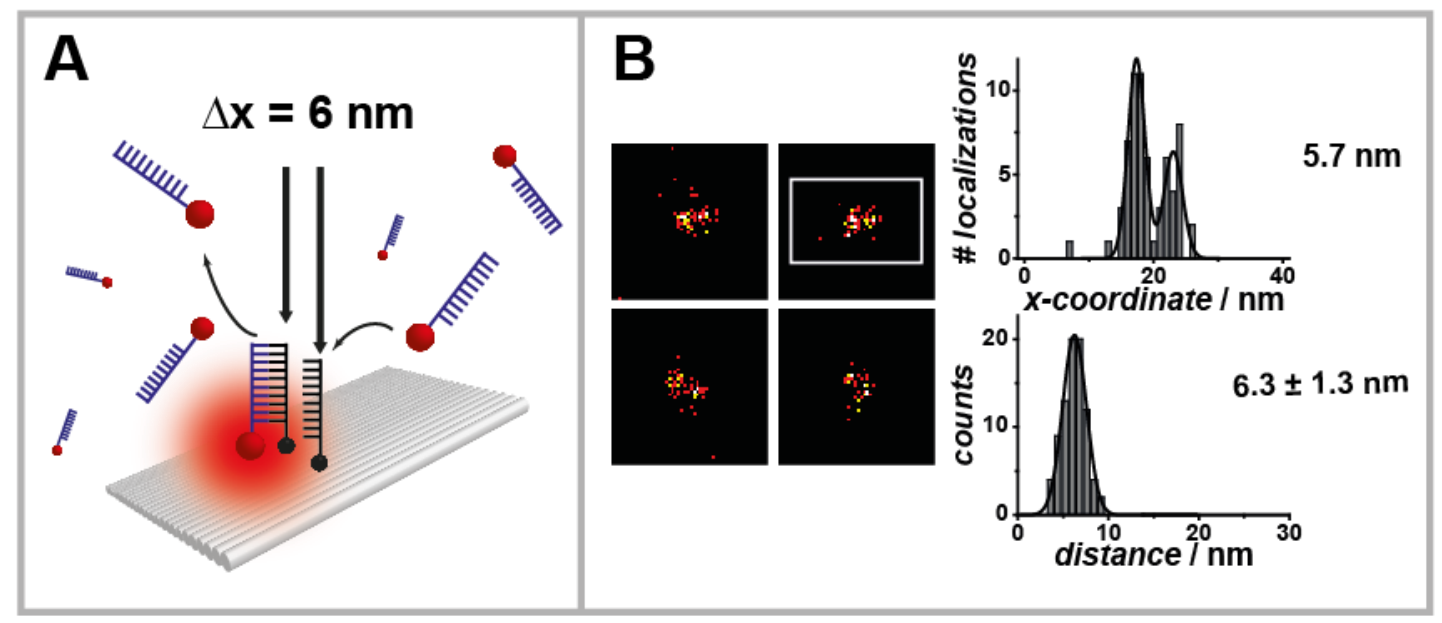

Figure 6 DNA-PAINT with ultra-high resolution: DNA Origami rectangle containing binding sites with a distance of $6 \mathrm{~nm}$ (A) and corresponding DNA-PAINT data resolving two binding sites at $6 \mathrm{~nm}$ separation (B) (adapted with permission from [39]).

\section{Other methods with specific binding}

Beside DNA-PAINT another method called uPAINT was published in 2010 by Giannone and coworkers [17]. It also improves the original PAINT by specific binding. Instead of DNA, dye labelled antibodies were used that continuously and sequentially bind molecules of interest on the cell surface. In addition to superresolution imaging, a tracking algorithm was used to generate dense diffusion maps of different target proteins on living cells.

Schoen et al. used STB to image chromosomal DNA in cells and amyloid fibrils by using low concentration of DNA-binding dyes that are activated due to the binding. This assay is called "binding-activated localization microscopy" (BALM) $[40,41]$. Simonson et al. imaged chromosomal DNA with a complementary approach and a localization analysis method called "gSHRImP" that makes use of intensity contrasts between images caused by sequential dye photobleaching [42].

The recent multi-target superresolution technique IRIS (image reconstruction by integrating exchangeable single-molecule localization) uses protein fragment-based probes that directly associate with and dissociate from their target without the need of any labelling [43]. This enables high-density labeling of the targets and thus a resolution enhancement when resolution is limited by the density of labeling. Figure 7 shows IRIS-images with increasing numbers of localization over time. 


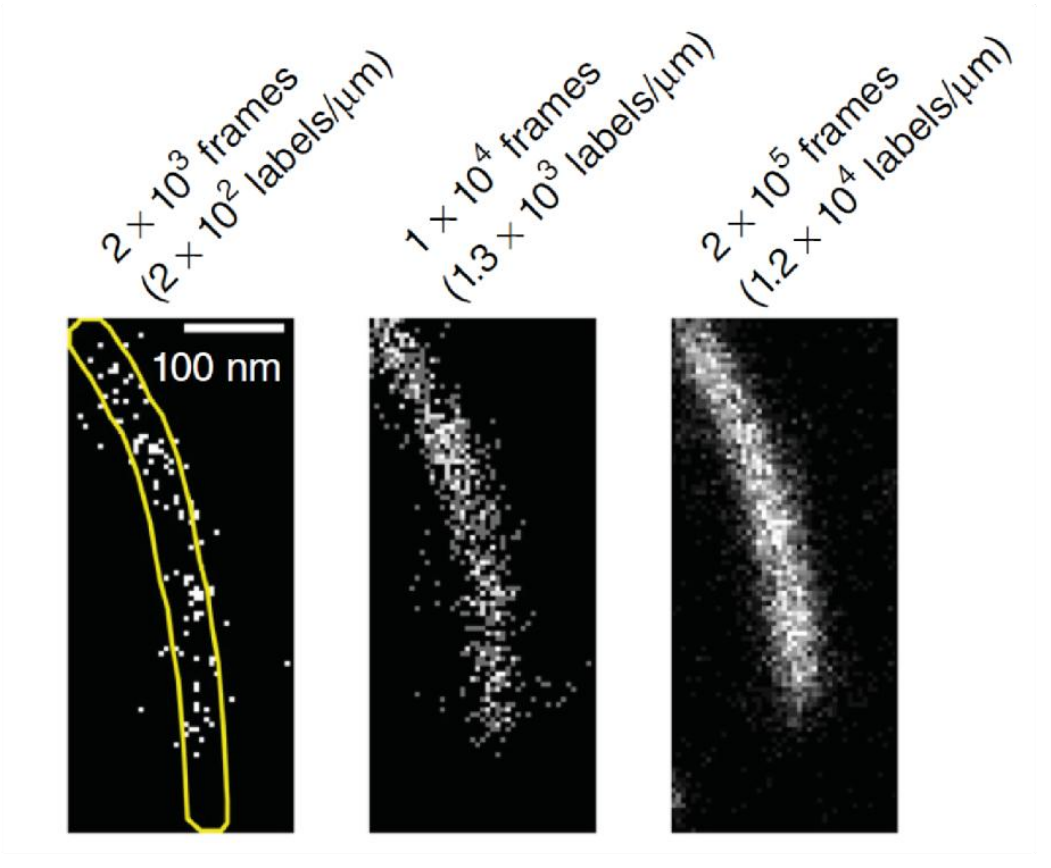

Figure 7 STB with IRIS: the figure shows different labeling densities of an actin filament at different acquisition times, using an Atto-488 labeled derivate of the actin binding peptide Lifeact that has a binding time of $0.4 \mathrm{~s}$ to the target [43] (data adapted with permission).

\section{Perspective}

STB passed through a short-term evolution. Within the last few years, the idea evolved and different variations are now in many ways competitive with other superresolution modalities. It is nowadays applicable for biological systems, for instance fluorescence in situ hybridization (FISH) with improved resolution and sensitivity [37]. Combinations with other superresolution fluorescence techniques as described above (photoswitching, STED, SHRImP) created more groundbreaking applications, making this method still straightforward to perform $[13,36,42]$. The enabled resolution has closed the gap to the FRET-length scale, and considerable potential for interdisciplinary applications is emerging [39].

It is desirable to further establish STB for the investigation of biological systems. One key question is how to extend the labeling possibilities. There has been a long history of developing dye labeling for fixed and living cells, but the abilities to label with DNA or possibly more degradation-resistant variants such as locked DNA have not yet been addressed. Alternative weak interactions such as used for IRIS [43] have a high potential, but it will require a larger palette to address every protein of interest which will include genetically engineered tags. The advantage of this approach is that no chemical manipulation of the target structure is necessary and the cumulated labeling density should be very high. Single-molecule-pull-down experiments $[44,45]$ could help to find the needed molecules and investigate their dissociation constants, which is very crucial for STB .The most important transient labeling methods for STB mentioned in this review are summarized in Figure 8A.

Figure $8 \mathrm{~B}$ shows a potential methods to label biological samples with the nucleic acid adapter required for DNA-PAINT Imaging. These methods have already been used for labeling with fixed dyes and the corresponding chemistry should principally be suitable for the labeling with DNA [46-51] as well. 

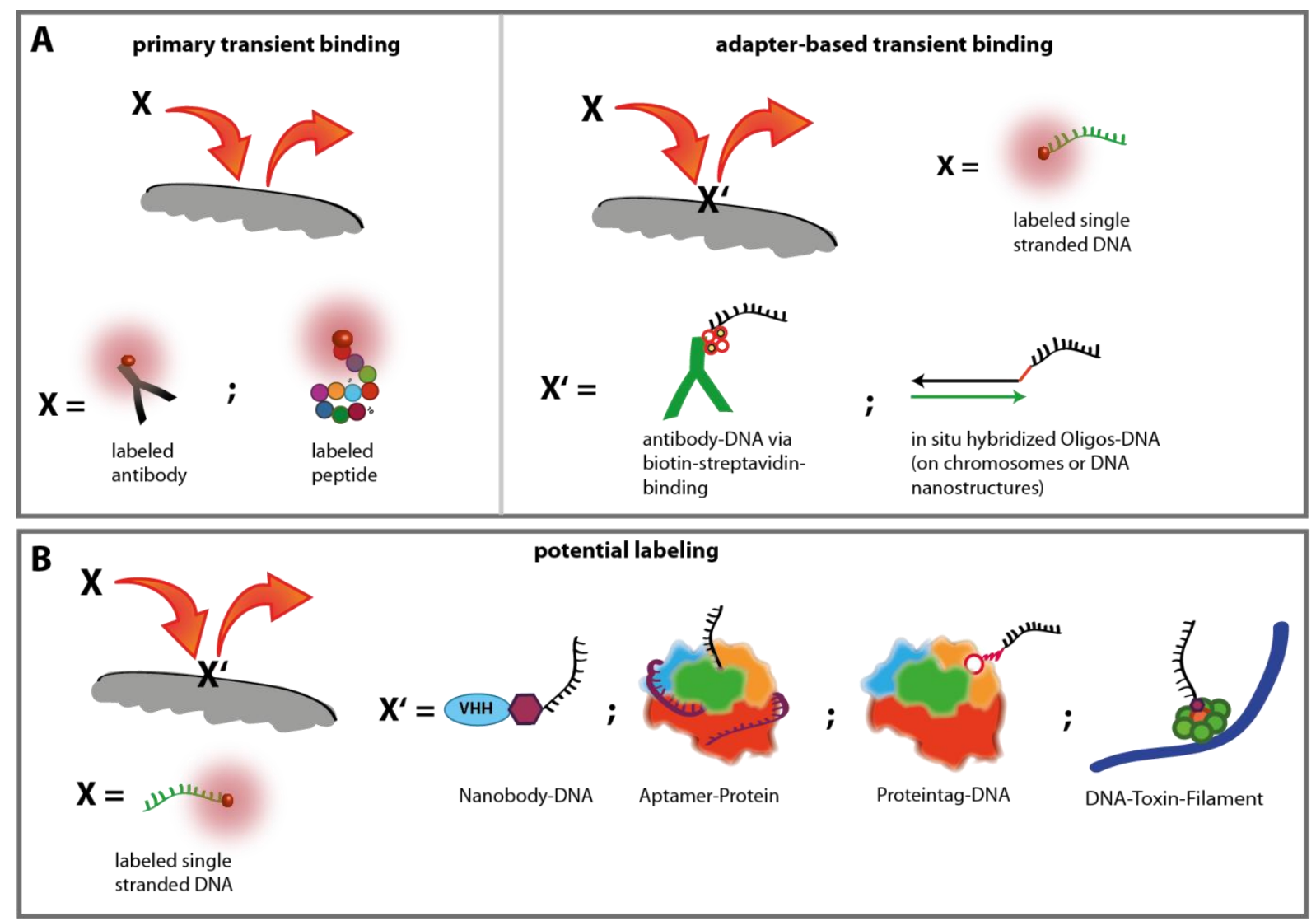

Figure 8 Labeling strategies for STB: established labeling strategies [11,14,17,35,37] (A) and potential labeling strategies for DNA PAINT (B). The established strategies exploit the specific binding of peptides or antibodies to the structure of interest without the need of any permanent labeling (primary binding) $[17,43]$. In contrast, structures of interest can be permanently labeled with DNA-docking sites for DNA PAINT (adapter based binding). So far this has been realized with in-situ-hybridization on DNA-nanostructures or chromosomes and with antibodies in cells [14,35,37]. As illustrated in (B) this principle could be extended to labeling methods that were originally developed for organic dyes. Examples include nanobodies [49], aptamers [52], protein tags like the SNAP-tag [50,51] or labeled phalloidin $[46,47]$ carrying a DNA-PAINT docking site instead of the dye.

Besides the labeling challenge, the restriction to TIRF or light sheet illumination is a second restriction that could especially be overcome using STB-probes that are only fluorescent in the bound form. This could be achieved with DNA hairpins carrying a quencher that is separated from the reporter dye upon binding. Already in the original PAINT and further works, substantial quenching of the unbound probes was exploited $[11,14,40]$. Further mechanisms in this direction could be dye self-quenching. With perfectly dark samples in the unbound states, thick samples could be imaged benefitting from the high photon counts and minimized photobleaching to the replenishment of the labels.

Overall, superresolution with transient binding is one of the most exciting emerging imaging modalities with unprecedented resolution, resistance to photobleaching and high labeling density. It is thus promising for many applications in bioimaging and nanotechnology.

\section{Acknowledgements}

This work was funded by the Braunschweig International Graduate School of Metrology B-IGSM and the DFG Research Training Group Grk1952/1 "Metrology for Complex Nanosystems", by a starting grant (SiMBA, EU 261162) of the European Research Council (ERC), by the National Science Foundation of China (21275109, 21205089, 91417301, 21475101) and the Humboldt Foundation for Professor Zhike He. 


\section{References}

1. Abbe E: Beiträge zur Theorie des Mikroskops und der mikroskopischen Wahrnehmung. Archiv für mikroskopische Anatomie 1873, 9:413-418.

2. Klar TA, Hell SW: Subdiffraction resolution in far-field fluorescence microscopy. Optics Letters 1999, 24:954956.

3. Betzig E, Patterson GH, Sougrat R, Lindwasser OW, Olenych S, Bonifacino JS, Davidson MW, LippincottSchwartz J, Hess HF: Imaging intracellular fluorescent proteins at nanometer resolution. Science 2006, 313:1642-1645.

4. Rust MJ, Bates M, Zhuang X: Sub-diffraction-limit imaging by stochastic optical reconstruction microscopy (STORM). Nat Methods 2006, 3:793-795.

5. Hess ST, Girirajan TP, Mason MD: Ultra-high resolution imaging by fluorescence photoactivation localization microscopy. Biophys J 2006, 91:4258-4272.

6. Heilemann M, van de Linde S, Schuttpelz M, Kasper R, Seefeldt B, Mukherjee A, Tinnefeld P, Sauer M: Subdiffraction-Resolution Fluorescence Imaging with Conventional Fluorescent Probes. Angew Chem Int Ed Engl 2008, 47:6172-6176.

7. Vogelsang J, Steinhauer C, Forthmann C, Stein IH, Person-Skegro B, Cordes T, Tinnefeld P: Make them Blink: Probes for Super-Resolution Microscopy. Chemphyschem 2010, 11:2475-2490.

8. Deschout H, Zanacchi FC, Mlodzianoski M, Diaspro A, Bewersdorf J, Hess ST, Braeckmans K: Precisely and accurately localizing single emitters in fluorescence microscopy. Nature methods 2014, 11:253-266.

9. Fernandez-Suarez M, Ting AY: Fluorescent probes for super-resolution imaging in living cells. Nat Rev Mol Cell Biol 2008, 9:929-943.

10. van de Linde S, Loschberger A, Klein T, Heidbreder M, Wolter S, Heilemann M, Sauer M: Direct stochastic optical reconstruction microscopy with standard fluorescent probes. Nature Protocols 2011, 6:9911009.

11. Sharonov A, Hochstrasser RM: Wide-field subdiffraction imaging by accumulated binding of diffusing probes. Proceedings of the National Academy of Sciences of the United States of America 2006, 103:18911-18916. (0

This was the publication that established the idea of superresolution microscopy by transient binding.

12. Kuo C, Hochstrasser RM: Super-resolution microscopy of lipid bilayer phases. Journal of the American Chemical Society 2011, 133:4664-4667.

13. Lew MD, Lee SF, Ptacin JL, Lee MK, Twieg RJ, Shapiro L, Moerner WE: Three-dimensional superresolution colocalization of intracellular protein superstructures and the cell surface in live Caulobacter crescentus. Proc Natl Acad Sci U S A 2011, 108:E1102-1110.

14. Jungmann R, Steinhauer C, Scheible M, Kuzyk A, Tinnefeld P, Simmel FC: Single-Molecule Kinetics and Super-Resolution Microscopy by Fluorescence Imaging of Transient Binding on DNA Origami. Nano Lett 2010, 10:4756-4761.

This was the first publication presenting DNA PAINT, the probably most popular realization of STB. It is also one of the first experiments that uses STB with target specific binding.

15. Thompson NL, Burghardt TP, Axelrod D: Measuring surface dynamics of biomolecules by total internal reflection fluorescence with photobleaching recovery or correlation spectroscopy. Biophysical journal 1981, 33:435.

16. Tokunaga M, Imamoto N, Sakata-Sogawa K: Highly inclined thin illumination enables clear single-molecule imaging in cells. Nat Methods 2008, 5:159-161.

17. Giannone G, Hosy E, Levet F, Constals A, Schulze K, Sobolevsky Al, Rosconi MP, Gouaux E, Tampe R, Choquet $D$, et al.: Dynamic Superresolution Imaging of Endogenous Proteins on Living Cells at UltraHigh Density. Biophysical Journal 2010, 99:1303-1310.

STB with target specific binding by antibodies was shown and used for a live-cell-application of combined superresolution and tracking.

18. Seidel CAM, Schulz A, Sauer MHM: Nucleobase-specific quenching of fluorescent dyes .1. Nucleobase oneelectron redox potentials and their correlation with static and dynamic quenching efficiencies. Journal of Physical Chemistry 1996, 100:5541-5553.

19. Rothemund PW: Folding DNA to create nanoscale shapes and patterns. Nature 2006, 440:297-302. 
20. Steinhauer C, Jungmann R, Sobey TL, Simmel FC, Tinnefeld P: DNA Origami as a Nanoscopic Ruler for Super-Resolution Microscopy. Angew Chem Int Ed Engl 2009, 48:8870-8873.

21. Schmied JJ, Gietl A, Holzmeister P, Forthmann C, Steinhauer C, Dammeyer T, Tinnefeld P: Fluorescence and super-resolution standards based on DNA origami. Nature Methods 2012, 9:1133-1134.

22. Schmied JJ, Raab M, Forthmann C, Pibiri E, Wunsch B, Dammeyer T, Tinnefeld P: DNA origami-based standards for quantitative fluorescence microscopy. Nature protocols 2014, 9:1367-1391.

23. Schmied JJ, Forthmann C, Pibiri E, Lalkens B, Nickels P, LiedI T, Tinnefeld P: DNA origami nanopillars as standards for three-dimensional superresolution microscopy. Nano letters 2013, 13:781-785.

24. Chen J, Bremauntz A, Kisley L, Shuang B, Landes CF: Super-Resolution mbPAINT for Optical Localization of Single-Stranded DNA. ACS applied materials \& interfaces 2013, 5:9338-9343.

25. linuma R, Ke Y, Jungmann R, Schlichthaerle T, Woehrstein JB, Yin P: Polyhedra Self-Assembled from DNA Tripods and Characterized with 3D DNA-PAINT. Science 2014.

3D-DNA-PAINT resolved the structure of novel DNA polyhedra that were difficult to image by other means - an illustrative example of the potential of $3 D$ superresolution by STB.

26. Deschamps J, Mund M, Ries J: 3D superresolution microscopy by supercritical angle detection. Optics express 2014, 22:29081-29091.

27. Derr ND, Goodman BS, Jungmann R, Leschziner AE, Shih WM, Reck-Peterson SL: Tug-of-war in motor protein ensembles revealed with a programmable DNA origami scaffold. Science 2012, 338:662-665.

28. Knudsen JB, Liu L, Bank Kodal AL, Madsen M, Li Q, Song J, Woehrstein JB, Wickham SFJ, Strauss MT, Schueder F, et al.: Routing of individual polymers in designed patterns. Nat Nano 2015, 10:892-898.

29. Lin C, Jungmann R, Leifer AM, Li C, Levner D, Church GM, Shih WM, Yin P: Submicrometre geometrically encoded fluorescent barcodes self-assembled from DNA. Nat Chem 2012, 4:832-839.

30. Cordes T, Vogelsang J, Steinhauer C, Stein I, Forthmann C, Gietl A, Schmied J, Acuna G, Laurien S, Lalkens B, et al.: Far-Field Nanoscopy with Conventional Fluorophores: Photostability, Photophysics, and Transient Binding. Springer Berlin Heidelberg; 2012:1-28.

31. Johnson-Buck A, Nangreave J, Kim DN, Bathe M, Yan H, Walter NG: Super-resolution fingerprinting detects chemical reactions and idiosyncrasies of single DNA pegboards. Nano letters 2013, 13:728-733.

32. Johnson-Buck A, Nangreave J, Jiang S, Yan H, Walter NG: Multifactorial modulation of binding and dissociation kinetics on two-dimensional DNA nanostructures. Nano letters 2013, 13:2754-2759.

33. Johnson-Buck A, Walter NG: Discovering anomalous hybridization kinetics on DNA nanostructures using single-molecule fluorescence microscopy. Methods 2014, 67:177-184.

34. Scheible MB, Pardatscher G, Kuzyk A, Simmel FC: Single molecule characterization of DNA binding and strand displacement reactions on lithographic DNA origami microarrays. Nano letters 2014, 14:16271633.

35. Jungmann R, Avendano MS, Woehrstein JB, Dai M, Shih WM, Yin P: Multiplexed 3D cellular superresolution imaging with DNA-PAINT and Exchange-PAINT. Nature methods 2014, 11:313-318.

This works extends DNA-PAINT for multiplexed imaging of cells with only a single laser line. Multiplexing is achieved by varying DNA sequences of docking and imager strands.

36. Beater S, Holzmeister P, Lalkens B, Tinnefeld P: Simple and aberration-free 4color-STED--multiplexing by transient binding. Optics express 2015, 23:8630-8638.

37. Beliveau BJ, Boettiger AN, Avendano MS, Jungmann R, McCole RB, Joyce EF, Kim-Kiselak C, Bantignies F, Fonseka $\mathrm{CY}$, Erceg J, et al.: Single-molecule super-resolution imaging of chromosomes and in situ haplotype visualization using Oligopaint FISH probes. Nature communications 2015, 6.

38. Beliveau BJ, Joyce EF, Apostolopoulos N, Yilmaz F, Fonseka CY, McCole RB, Chang Y, Li JB, Senaratne TN, Williams BR: Versatile design and synthesis platform for visualizing genomes with Oligopaint FISH probes. Proceedings of the National Academy of Sciences 2012, 109:21301-21306.

39. Raab M, Schmied JJ, Jusuk I, Forthmann C, Tinnefeld P: Fluorescence microscopy with 6 nm resolution on DNA origami. Chemphyschem 2014, 15:2431-2435.

This work demonstrates the resolving power of DNA-PAINT reaching the lengthscale of FRET measurements thus closing the resolution gap.

40. Schoen I, Ries J, Klotzsch E, Ewers H, Vogel V: Binding-Activated Localization Microscopy of DNA Structures. Nano Letters 2011, 11:4008-4011.

41. Ries J, Udayar V, Soragni A, Hornemann S, Nilsson KP, Riek R, Hock C, Ewers H, Aguzzi AA, Rajendran L: Superresolution imaging of amyloid fibrils with binding-activated probes. ACS chemical neuroscience 2013, 4:1057-1061.

42. Simonson PD, Rothenberg E, Selvin PR: Single-molecule-based super-resolution images in the presence of multiple fluorophores. Nano letters 2011, 11:5090-5096. 
43. Kiuchi T, Higuchi M, Takamura A, Maruoka M, Watanabe N: Multitarget super-resolution microscopy with high-density labeling by exchangeable probes. Nat Meth 2015, 12:743-746.

Using different transiently binding probes, cell-imaging via STB was shown. Avoiding permanent binding, very high accumulated labeling densities were demonstrated for different target structures.

44. Jain A, Liu R, Ramani B, Arauz E, Ishitsuka Y, Ragunathan K, Park J, Chen J, Xiang YK, Ha T: Probing cellular protein complexes using single-molecule pull-down. Nature 2011, 473:484-488.

45. Tinnefeld P: Protein-protein interactions: Pull-down for single molecules. Nature 2011, 473:461-462.

46. Wulf E, Deboben A, Bautz FA, Faulstich H, Wieland T: Fluorescent phallotoxin, a tool for the visualization of cellular actin. Proceedings of the National Academy of Sciences 1979, 76:4498-4502.

47. Barak LS, Yocum RR, Nothnagel EA, Webb WW: Fluorescence staining of the actin cytoskeleton in living cells with 7-nitrobenz-2-oxa-1,3-diazole-phallacidin. Proceedings of the National Academy of Sciences 1980, 77:980-984.

48. Panchuk-Voloshina N, Haugland RP, Bishop-Stewart J, Bhalgat MK, Millard PJ, Mao F, Leung W-Y, Haugland RP: Alexa Dyes, a Series of New Fluorescent Dyes that Yield Exceptionally Bright, Photostable Conjugates. Journal of Histochemistry \& Cytochemistry 1999, 47:1179-1188.

49. Ries J, Kaplan C, Platonova E, Eghlidi H, Ewers H: A simple, versatile method for GFP-based superresolution microscopy via nanobodies. Nat Meth 2012, 9:582-584.

50. Jones SA, Shim S-H, He J, Zhuang X: Fast, three-dimensional super-resolution imaging of live cells. Nat Meth 2011, 8:499-505.

51. Klein T, Loschberger A, Proppert S, Wolter S, van de Linde S, Sauer M: Live-cell dSTORM with SNAP-tag fusion proteins. Nat Methods 2011, 8:7-9.

52. Opazo F, Levy M, Byrom M, Schafer C, Geisler C, Groemer TW, Ellington AD, Rizzoli SO: Aptamers as potential tools for super-resolution microscopy. Nature methods 2012, 9:938-939. 